\title{
5 The Expressive Power of Contextualist Truth
}

\author{
Julien Murzi and Lorenzo Rossi
}

The truth predicate is often argued to be naïve, in the sense a sentence $\varphi$ and its truth-ascription ' $\varphi$ is true', in symbols $\operatorname{Tr}(\ulcorner\varphi\urcorner)$, are in some way equivalent (in all non-opaque contexts). ${ }^{1}$ Some authors require that $\varphi$ and $\operatorname{Tr}(\ulcorner\varphi\urcorner)$ be interderivable (from possibly open assumptions), and thus obey the following introduction and elimination rules: ${ }^{2}$

$$
\frac{\varphi}{\operatorname{Tr}(\ulcorner\varphi\urcorner)} \operatorname{Tr}-\mathrm{I} \quad \frac{\operatorname{Tr}(\ulcorner\varphi\urcorner)}{\varphi} \operatorname{Tr}-\mathrm{E}
$$

Other authors go further and demand that the truth predicate obey the full T-Schema:

$$
\operatorname{Tr}(\ulcorner\varphi\urcorner) \leftrightarrow \varphi
$$

or the so-called transparency requirement, according to which any sentence $\varphi$ is intersubstitutable salva veritate with $\operatorname{Tr}(\ulcorner\varphi\urcorner)$, in all nonopaque contexts.

It has been forcefully argued that naïveté is required in order to account for crucial and non-eliminable uses of the truth predicate, involving blind ascriptions (such as 'Everything Lois says is true'), infinitary generalizations (such as 'All theorems of Peano Arithmetic are true'), and their combinations (Field, 2008; Beall, 2009; Horsten, 2012). Call this argument the Argument for Naïveté. Since all forms of naïveté are incompatible with classical logic (given a modicum of syntax theory, the argument can be taken to establish that, in order to fulfil its inferential role, the truth predicate requires a suitable nonclassical logic.

To be sure, the Argument for Naïveté does not settle which form of naïveté is best suited to underwrite the expressive role of 'true'. Some authors (see e.g. Field, 2008 and Beall, 2009) argue that naïve rules such as Tr-I and Tr-E do not suffice to model reasonings involving blind ascriptions and infinitary generalizations in certain embedded contexts, such as the antecedent of a conditional claim. For this reason, they propose stronger forms of naïveté, such as the T-Schema 
(in the presence of a suitably strong logic of the conditional), or transparency. Call this the Argument for Transparency.

In this chapter, we argue that both the Argument for Transparency and the Argument for Naïveté are fundamentally misguided. We first show, contra Field, that the expressive role of 'true' does not require transparency: principles such as $\mathrm{Tr}-\mathrm{I}$ and $\mathrm{Tr}-\mathrm{E}$ are indeed sufficient. However, we also argue, contra an argument offered by Picollo and Schindler (2018) that principles such as Tr-I and Tr-E are necessary: theories that do not validate similar principles irredeemably cripple the expressive role of 'true' (\$5.1). We then point to some fully classical, non-naïve theories of truth featuring versions of Tr-I and Tr-E that are strong enough to validate arguments involving blind ascriptions and infinitary generalizations and yet weak enough to avoid paradox-driven triviality. In particular, we argue that contextualist theories of truth, despite being fully classical, feature expressively adequate, context-shifting versions of the naïve truth introduction and elimination rules $(\S 5.2)$. We conclude that the expressive role of the truth predicate requires neither transparency nor naïveté.

\subsection{The Inferential Role of Truth and Non-Classical Logic}

We begin by considering simple, unembedded cases of agreement and disagreement (\$5.1.1) and then move to more complex, embedded such cases, and to Field's Argument for Transparency (\$5.1.2). We suggest that unembedded and embedded cases alike provide strong evidence for the interderivability of $\varphi$ and ' $\varphi$ is true', at least for languages, such as English, that are rich enough to express so-called contingent liars. However, we also argue that, pace Field, embedded cases of agreement and disagreement fail to establish that the truth predicate ought to be 'transparent'-i.e. transparency is not necessary for the inferential role of 'true'.

\subsubsection{Simple Agreement and Disagreement}

It is often argued that simple cases of agreement and disagreement motivate the adoption of naïve introduction and elimination rules for 'true' and, in turn, of a non-classical theory of truth. But this argument is itself naïve, or so the classical theorist might argue.

\section{The Argument for Naïveté}

Consider the following argument-call it AgreEment:

(1) All the theorems of Peano Arithmetic are true;

(2) If $\ulcorner\varphi\urcorner$ is a theorem of Peano Arithmetic, then $\operatorname{Tr}(\ulcorner\varphi\urcorner)$; 
(3) $\ulcorner\varphi\urcorner$ is a theorem of Peano Arithmetic;

(4) $\operatorname{Tr}(\ulcorner\varphi\urcorner)$;

(5) $\varphi$.

Here, thanks to the truth-predicate, we move from an expression of agreement with a given set of claims to the actual assertion of one of these claims. The steps from (1) to (4) seem unassailable: (1) and (3) are assumptions, (2) follows from (1) by universal instantiation, and (4) follows from (2) and (3) by modus ponens. To be sure, principles such as universal instantiation and modus ponens might be called into question (see e.g. McGee, 1985). However, given our focus on truth, we bracket aside any non-truth-theoretic qualms one might have with classical rules. Then, the only potentially suspicious step is the one from (4) to (5), which employs Tr-E.

A converse argument illustrates how one can move from an expression of disagreement with a given set of claims to the actual assertion of the negation of one of those claims-call this Disagreement:

(6) Everything Lois said yesterday is not true;

(7) If Lois said $\ulcorner\psi\urcorner$ yesterday, then $\neg \operatorname{Tr}(\ulcorner\psi\urcorner)$;

(8) Yesterday Lois said $\ulcorner\psi\urcorner$;

(9) $\neg \operatorname{Tr}(\ulcorner\psi\urcorner)$;

(10) $\neg \psi$.

As above, the steps from (6) to (9) appear completely unproblematic: (6) and (8) are assumptions, (7) follows from (6) by universal instantiation, and (9) follows from (7) and (8) by modus ponens. Again, the only potentially controversial step is the one from (9) to (10), which employs Tr-I (given contraposition).

Speeches such as AgreEment and Disagreement prima facie suggest that the expressive role of 'true' requires Tr-I and Tr-E. After all, the argument goes, it is difficult to see how these reasonings can be run without these principles. Without Tr-E, one can at best use AgreEMENT to establish $\operatorname{Tr}(\ulcorner\varphi\urcorner)$ from the premises that the theorems of Peano Arithmetic are true and that $\varphi$ is a theorem of Peano Arithmetic. But this falls short of establishing $\varphi$. Similar considerations hold for DisagREEMENT. In order to fulfil its expressive role, the truth predicate must obey both Tr-I and Tr-E and, for this reason, any expressively adequate theory of truth must be non-classical, or so non-classical theorists argue (Field, 2008; Beall, 2009; Horsten, 2012).

\section{Contingent Liars}

It might be objected that the argument at best establishes the validity of certain instances of Tr-E and Tr-I-namely, those occurring in 
compelling instances of Agreement and Disagreement. For instance, consider the following (non-schematic) instance of AGREEMENT:

(1) All the theorems of Peano Arithmetic are true;

(2*) If $\ulcorner 2+2=4\urcorner$ is a theorem of Peano Arithmetic, then $\operatorname{Tr}(\ulcorner 2+2=4\urcorner)$;

( $\left.3^{\star}\right)\ulcorner 2+2=4\urcorner$ is a theorem of Peano Arithmetic;

(4*) $\operatorname{Tr}(\ulcorner 2+2=4\urcorner)$;

(5*) $2+2=4$.

And consider the following (non-schematic) instance of DisAgREEMENT:

(6) Everything Lois said yesterday is not true;

$\left(7^{\star}\right)$ If Lois said $\ulcorner$ grass is red $\urcorner$ yesterday, then $\neg \operatorname{Tr}(\ulcorner$ grass is $\operatorname{red}\urcorner)$;

$\left(8^{\star}\right)$ Yesterday Lois said $\ulcorner$ grass is red $\urcorner$;

$\left(9^{\star}\right) \neg \operatorname{Tr}(\ulcorner$ grass is $\operatorname{red}\urcorner)$;

$\left(10^{\star}\right)$ It is not the case that grass is red.

Both of the above arguments arguably provide good evidence for the corresponding instances of Tr-E and Tr-I. However, the classical theorist might insist, such instances fall short of justifying the schematic arguments Agreement and DisAgreEment-and hence fall short of justifying full Tr-E and Tr-I and the consequent abandonment of classical logic. There are two related reasons for this.

For one thing, virtually every classical theory of truth will allow one to infer $2+2=4$ from $\operatorname{Tr}(\ulcorner 2+2=4\urcorner)$, or $\neg(0=1)$ from $\neg \operatorname{Tr}(\ulcorner 0=1\urcorner))^{3,4}$ For another, virtually every classical theory of truth will disallow applications of Tr-I and Tr-E to paradoxical sentences, such as Liar and Curry sentences. However, and this is the crucial point, instances of AGREEMENT and DisAGREEMENT involving such sentences do not constitute convincing evidence to accept Tr-I and Tr-E, or at least so the classical theorist might argue. ${ }^{5}$ To see this, let $\lambda$ be a Liar sentence (that is, a sentence equivalent to its own negated truth-ascription $\neg \operatorname{Tr}(\ulcorner\lambda\urcorner)$ ), and consider the following instance of DisAGREEMENT:

(6) Everything Lois said yesterday is not true;

$\left(7^{\star \star}\right)$ If Lois said $\ulcorner\lambda\urcorner$ yesterday, then $\neg \operatorname{Tr}(\ulcorner\lambda\urcorner)$;

$\left(8^{\star \star}\right)$ Yesterday Lois said $\ulcorner\lambda\urcorner$;

$\left(9^{\star \star}\right) \neg \operatorname{Tr}(\ulcorner\lambda\urcorner)$;

$\left(10^{\star \star}\right) \neg \lambda$.

Classical theorists might insist that is not at all clear whether it is desirable to infer $\neg \lambda$, that is $\operatorname{Tr}(\ulcorner\lambda\urcorner)$, from $\neg \operatorname{Tr}(\ulcorner\lambda\urcorner)$. As a result, it is not at all clear whether the corresponding instance of Tr-I (which is required to move from $\left(9^{\star \star}\right)$ to $\left.\left(10^{\star \star}\right)\right)$ is justified. 


\section{2}

Julien Murzi and Lorenzo Rossi

Putting the two halves of her argument together, the classical theorist can claim that she can recover all the uncontroversial instances of AGREEMENT and DisAgreEMENT while insisting that the ones involving paradoxical sentences fail to justify full Tr-I and Tr-E. For instance, the classical theorist might insist that she only accepts instances of argument forms such as Agreement and Disagreement that are clearly safe-e.g. instances involving sentences involving no semantic vocabulary, or truth iterations of such sentences.

The foregoing rejoinder, though, presupposes that it is always clear whether an instance of Tr-E or Tr-I is paradoxical, or unsafe. But this is actually not the case. As Kripke (1975) famously pointed out, whether a sentence is paradoxical can depend on contingent facts. For instance, suppose Lois says that what the lady on TV with the red dress says is not true and that, as a matter of fact, she is the lady on TV with the red dress. Then, Lois' utterance is paradoxical, but, one can persuasively argue, the corresponding versions of AGREEMENT and DisAGREEMENT are perfectly acceptable, as is arguably shown by the following instance of DISAGREEMENT:

(6) Everything Lois says is not true;

$\left(7^{\star \star \star}\right)$ If Lois says $\ulcorner$ what the lady on TV with the red dress says is not true $\urcorner$, then $\neg \operatorname{Tr}(\ulcorner$ what the lady on TV with the red dress says is not true $\urcorner$ );

$\left(8^{\star \star \star}\right)$ Lois says $\ulcorner$ what the lady on TV with the red dress says is not true 7

$\left(9^{\star \star \star}\right) \neg \operatorname{Tr}(\ulcorner$ what the lady on TV with the red dress says is not true $\urcorner)$; $\left(10^{\star \star \star}\right)$ It is not the case that what the lady on TV with the red dress says is not true.

Now, whether 'what the lady on TV with the red dress says is not true' is paradoxical depends on the contingent facts, i.e. on whether the speaker is the lady on TV with the red dress. But, then, there are countless perfectly harmless instances of $(6)-\left(10^{\star \star \star}\right)$. And, it seems plausible to maintain, it would be unduly restrictive to disallow them all. As Field puts it, this would irredeemably 'cripple' ordinary reasoning. It would seem, then, that the inferential role of the truth predicate indeed requires, just like non-classical theorists maintain, that 'true' satisfies principles that validate the interderivability of $\varphi$ and the claim that $\varphi$ is true, such as Tr-E and Tr-I.

Field further argues that embedded uses of 'true' (as in the antecedent of conditionals) actually require that truth be transparent, i.e. that the truth predicate satisfies the intersubstitutivity of $\operatorname{Tr}(\ulcorner\varphi\urcorner)$ and $\varphi$ in all non-opaque contexts. We disagree. Before we say why, we first review Field's Argument for Transparency. 


\subsubsection{Embedded Truth-Ascriptions and the Argument for Transparency}

We present Field's Argument for Transparency and argue that it misses its target: the embedded uses of 'true' Field points to can be adequately modelled by Tr-E and Tr-I. We then consider a recent argument by Lavinia Picollo and Thomas Schindler, which can be interpreted as showing that Tr-E is sufficient to model the expressive uses of 'true', and find it wanting.

\section{Field's Argument}

According to Field (2008), more complex arguments involving embedded truth-ascriptions show that the truth predicate must obey full transparency, and not merely Tr-E and Tr-I. He writes:

Talk of truth isn't just a means of expressing agreement and disagreement, for the same reason that talk of goodness isn't just a means of expressing approval and disapproval: 'true', like 'good', occurs in embedded contexts (contexts embedded more deeply than a negation). In particular, 'true' is used inside conditionals. And in order for it to serve its purpose, it needs to be well-behaved there: inside conditionals as in unembedded contexts, 'true' needs to serve as a device of infinite conjunction or disjunction.... Suppose I can't remember exactly what was in the Conyers report on the 2004 election, but say.

(11) If everything that the Conyers report says is true, then the 2004 election was stolen.

Suppose that what the Conyers report says is $\varphi_{1}, \ldots, \varphi_{n}$. Then relative to this last supposition, (11) better be equivalent to

(12) If $\varphi_{1}, \ldots, \varphi_{n}$, then the 2004 election was stolen.

And this requires $\operatorname{True}(\ulcorner\varphi\urcorner)$ to be intersubstitutable with $\varphi$ even when $\varphi$ is the antecedent of a conditional.

(Field, 2008, pp. 109-110; the original numbering has been adapted to ours)

However, we argue, pace Field, embedded truth-ascriptions do not show that truth has to be fully transparent in order to fulfil its inferential role.

\section{Tr-E and Tr-I Suffice}

Let $\mathrm{C}(\ulcorner\varphi\urcorner)$ and $\sigma$ be shorthand for, respectively, 'The Conyers report says that $\varphi$ ' and 'The election was stolen'. Let $\chi$ be the conjunction of 
all the claims in the Conyers report. Consider the following two sentences:

(13) $\forall x(\mathrm{C}(x) \rightarrow \operatorname{Tr}(x)) \rightarrow \sigma$;

(14) $\chi \rightarrow \sigma$.

We now show that (13) and (14) are interderivable via Tr-E and Tr-I, together with $C(\ulcorner\chi\urcorner)$ and suitable assumptions on how to formalise the sentence concerning what the report says.

We first show that (13) follows from (14) and $C(\ulcorner\chi\urcorner)$, given Tr-E:

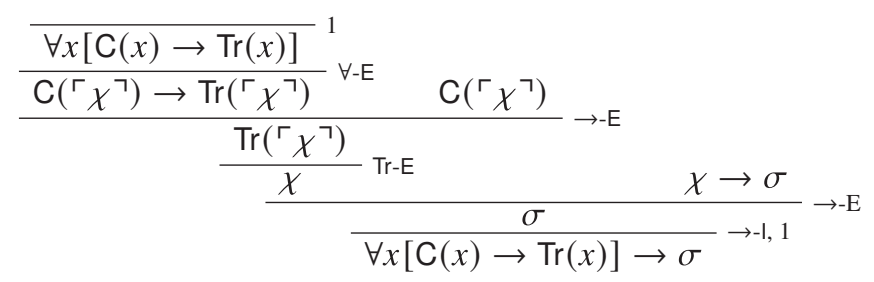

We then establish that (14) follows from (13), given Tr-I and $\forall x\left(\mathrm{C}(x) \leftrightarrow x=\left\ulcorner\varphi_{1}\right\urcorner \vee \ldots \vee x=\left\ulcorner\varphi_{n}\right\urcorner\right)$, which expresses the fact that the Conyers report says exactly that $\chi$. Let $\zeta$ be a shorthand for $\mathrm{C}(x) \leftrightarrow x=\left\ulcorner\varphi_{1}\right\urcorner \vee \ldots \vee x=\left\ulcorner\varphi_{n}\right\urcorner$. We can then reason thus:

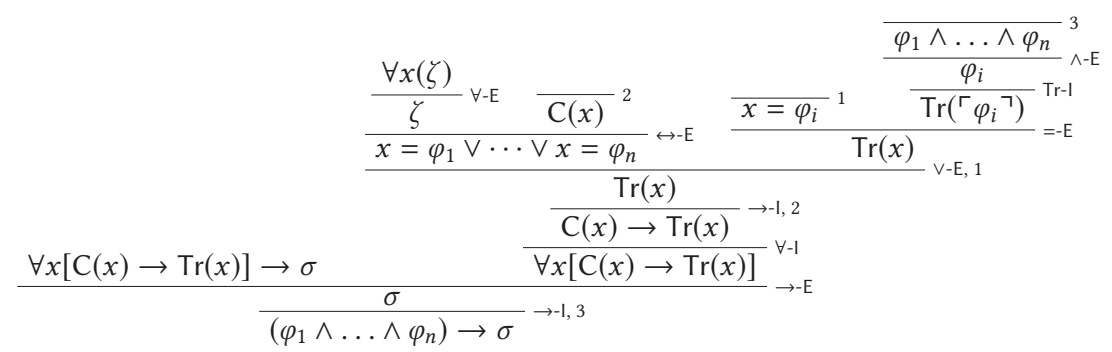

The line labelled ' $\mathrm{V}-\mathrm{E}, 1$ ' is a condensed way to indicate $n$-many uses of the rule of disjunction elimination (and the corresponding assumption discharges). Writing this step in full, we should have first applied disjunction elimination to $x=\left\ulcorner\varphi_{1}\right\urcorner \vee \ldots \vee x=\left\ulcorner\varphi_{n}\right\urcorner$, then to $x=\left\ulcorner\varphi_{2}\right\urcorner \vee \ldots \vee x=$ $\left\ulcorner\varphi_{n}\right\urcorner$, and so on, until we reach $x=\left\ulcorner\varphi_{n-1}\right\urcorner \vee x=\left\ulcorner\varphi_{n}\right\urcorner$. We've omitted the full steps and abused notation in the manner indicated for readability's sake.

The situation is exactly parallel for cases of embedded disagreement. Letting $v$ be the disjunction of all the claims in the Conyers report (so that $\neg v$ amounts to the conjunction of all the negated claims in the report), consider the two following sentences: ${ }^{6}$

(15) $\forall x(\mathrm{C}(x) \rightarrow \neg \operatorname{Tr}(x)) \rightarrow \neg \sigma$;

(16) $\neg v \rightarrow \neg \sigma$. 
We first show that (15) follows from (16) and $C(\ulcorner v\urcorner)$, given Tr-I: ${ }^{7}$

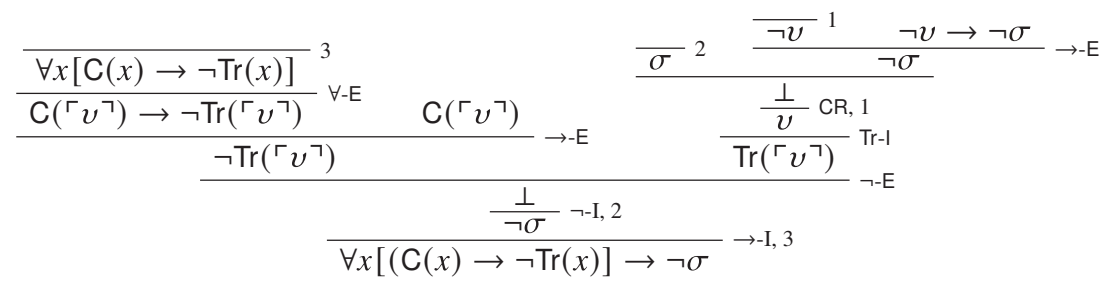

We then show that (16) follows from (15) and $\forall x(\zeta)$, given $\operatorname{Tr}-\mathrm{E}$. For every $\varphi_{i} \in\left\{\varphi_{1}, \ldots, \varphi_{n}\right\}$, call $\mathcal{D}_{i}$ the derivation of $\neg \operatorname{Tr}\left(\left\ulcorner\varphi_{i}\right\urcorner\right)$ from the open assumption $\neg \varphi_{i}$ via Tr-E: 8

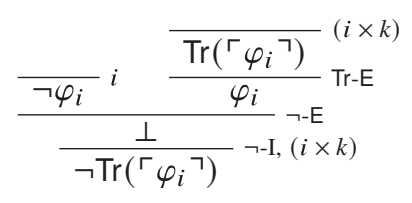

We can now derive

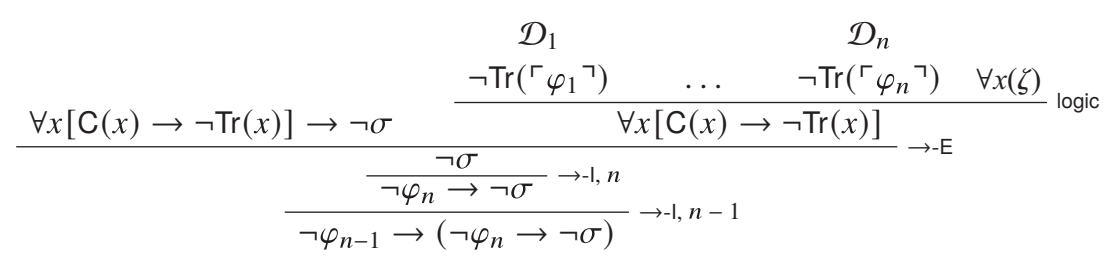

$$
\begin{aligned}
& \frac{\neg \varphi_{1} \rightarrow\left(\ldots\left(\neg \varphi_{n-1} \rightarrow\left(\neg \varphi_{n} \rightarrow \neg \sigma\right)\right) \ldots\right)}{\neg v \rightarrow \neg \sigma} \text { logic }
\end{aligned}
$$

which completes our proof. The upper inference line labelled 'logic' is established exactly as above, while the lower one corresponds to multiple uses of the import-export and De Morgan laws.

It might be objected that our reconstruction of the interderivability between (13) and (14), plus auxiliary assumpions, is unacceptable because it uses Tr-E and Tr-I together with $\rightarrow-I$ and $\rightarrow-E$, and these four rules, if taken unrestrictedly, yield triviality. ${ }^{9}$ On the other hand, the objection continues, showing (13) and (14) to be interderivable 'via transparency' does not risk triviality: there are transparent and nontrivial theories of truth (such as Field's own theory) where (13) and (14) are interderivabile, but where our reconstruction is not available, since one of $\rightarrow-I$ and $\rightarrow-E$ is not unrestrictedly valid. Therefore, the objection concludes, the Argument for Transparency still stands: we need transparency to non-trivially model the equivalence between (13) and (14).

We find the objection mistaken, for at least two reasons. First, the fact that a natural piece of reasoning is classically inconsistent is not by itself 
an objection against the principles employed in the reasoning (a fortiori for theorists who are in general open to revise classical logic). Consider again Agreement and Disagreement. They, too, are pieces of reasoning which employ classical logic and naïve truth-theoretical principles in order to motivate Tr-I and Tr-E, and hence the need for a theory which validates them. Entirely analogously, our reconstruction of the interderivability between (13) and (14) can be taken to motivate Tr-I and Tr-E, or relevantly similar principles, and the need for a suitable theory that validates those principles. Second, as we will see, contextualism does provide a classical theory where versions of Tr-I and Tr-E are available, thus allowing us to recover our reconstruction of the equivalence between (13) and (14).

Summing up, the above arguments show that, pace Field, embedded cases of agreement and disagreement such as the above ones don't require truth to be transparent. Pace Picollo and Schindler, however, such cases still require that the truth predicate obey both Tr-E and Tr-I, or suitably related principles.

\section{Picollo and Schindler on Tr-E}

In a recent paper, Picollo and Schindler (2018) offer arguments that can be interpreted as showing that truth-elimination principles such as Tr-E suffice for the truth predicate to serve its expressive purposes. One of their arguments employs a result of Halbach (1999), to the effect that truth-theoretical generalizations (that is sentences of the form $\forall x(\varphi(x) \rightarrow \operatorname{Tr}(x))$ ) have the same truth-free consequences as the collection of their instances (that is all the instances of the schema $\varphi(\ulcorner\psi\urcorner) \rightarrow \psi$ ) in (suitably expressive) classical theories closed under Tr-I and Tr-E, where, crucially, the application of the latter rules is restricted to truth-free sentences. Picollo and Schindler observe that Halbach's result can also be proven if one only assumes closure under Tr-E alone (again, this principle needs to be restricted to truth-free sentences). ${ }^{10}$ They subsequently turn to Field's argument concerning embedded agreement and disagreement, and argue that truth-introduction principles are not required. As they put it:

there is an alternative and easy way to deal with the [embedded agreement and disagreement] case that only involves an elimination principle. For we can express (14) with a simple generalisation of the form $\forall x(\varphi(x) \rightarrow \operatorname{Tr}(x))$, instead of (13). Let $\varphi(x)$ be the predicate ' $x$ is the unique sentence obtained by concatenating the conjunction of the $\mathrm{C}(x)$ with $\rightarrow \sigma^{\prime}$. Then, we can choose $[\forall x(\varphi(x) \rightarrow \operatorname{Tr}(x))]$ to express (14): by [Picollo and Schindler's strengthening of Halbach's result], in any classical theory (that contains enough syntax theory to prove basic facts about concatenation) [closed under Tr-E restricted to sentences not containing the truth predicate] we can derive the 
latter from the former, relative to the assumption that what [is written in the report] is exactly $\varphi_{1}, \ldots, \varphi_{n}$.

(Picollo and Schindler, 2018, p. 913, numbering and notation have been adapted to ours)

To exemplify, Picollo and Schindler suggest to interpret

(11) If everything that the Conyers report says is true, then the 2004 election was stolen.

as follows. First, consider all the things that the Conyers report says, namely $\varphi_{1}, \ldots, \varphi_{n}$. Then, consider the sentence $\varphi_{1} \wedge \ldots \wedge \varphi_{n} \rightarrow \sigma$, which says that if $\varphi_{1} \wedge \ldots \wedge \varphi_{n}$, then the election was stolen. Now consider the predicate 'results from concatenating $\varphi_{1} \wedge \ldots \wedge \varphi_{n}$ with $\rightarrow \sigma$ '. Of course, there is exactly one sentence satisfying such a predicate, namely $\varphi_{1} \wedge \ldots \wedge \varphi_{n} \rightarrow \sigma$. Such a sentence can now be used to express (11) as a truth-theoretical generalisation, namely

$\left(11^{+}\right)$Every sentence that results from concatenating $\varphi_{1} \wedge \ldots \wedge \varphi_{n}$ with $\rightarrow \sigma$ is true.

Picollo and Schindler therefore suggest to express (11), a sentence of the form $\forall x(\varphi(x) \rightarrow \operatorname{Tr}(x)) \rightarrow \psi$, as a sentence of the form $\forall x(\chi(x) \rightarrow \operatorname{Tr}(x))$. Crucially, the interderivability of sentences such as (11) and $\left(11^{+}\right)$only requires Tr-E.

Picollo and Schindler's strategy fails to convince, however, for at least two reasons. First, the applicability of their argument is undermined by the restriction of Tr-E to truth-free sentences. To see this, notice that while it is consistent for (sufficiently expressive) classical base theories to be closed under the unrestricted rule Tr-E (for examples, see Friedman and Sheard, 1987 and Halbach, 2011, Ch. 14), no classical base theory can be closed under the unrestricted Tr-E together with the assumption $\forall x\left(\mathrm{C}(x) \leftrightarrow x=\left\ulcorner\varphi_{1}\right\urcorner \vee \ldots \vee x=\left\ulcorner\varphi_{n}\right\urcorner\right)$, for $\mathrm{C}(x)$ an arbitrary monadic formula of the language including the truth predicate. For one can then take the latter formula to be $\forall x(\operatorname{Tr}(x) \leftrightarrow x=\ulcorner\lambda\urcorner)$, for $\lambda$ a Liar sentence, thus deriving $\operatorname{Tr}(\ulcorner\lambda\urcorner) \leftrightarrow\ulcorner\lambda\urcorner=\ulcorner\lambda\urcorner$ (by universal instantiation), and then $\operatorname{Tr}(\ulcorner\lambda\urcorner)$ (by modus ponens and the logic of identity), and finally $\neg \operatorname{Tr}(\ulcorner\lambda\urcorner$ ) (by $\operatorname{Tr}-E$ and the definition of $\lambda$ ), thus proving a contradiction. ${ }^{11}$

Second, it is not clear what, if not a covert use of Tr-I, can possibly justify the choice of $\varphi(x)$ in the formula $\forall x(\varphi(x) \rightarrow \operatorname{Tr}(x))$ in Picollo and Schindler's argument. They suggest to use $\forall x(\varphi(x) \rightarrow \operatorname{Tr}(x))$ in lieu of $\forall x(\mathrm{C}(x) \rightarrow \operatorname{Tr}(x)) \rightarrow \sigma$, where $\varphi(x)$ actually yields $\chi \rightarrow \sigma$ (relative to the assumption that what is written in the report is exactly $\left.\varphi_{1}, \ldots, \varphi_{n}\right)$. 
More precisely, we need some device for replacing a truth-theoretical generalisation in the antecedent of a conditional with its instances. But what justifies such a replacement, i.e. Picollo and Schindler's use of $\forall x(\varphi(x) \rightarrow \operatorname{Tr}(x))$ ? Picollo and Schindler do not address this question. Yet, derivations such as the derivation of (14) from (13) strongly suggest that the obvious answer is: a use of Tr-I! But such an answer is clearly precluded to Picollo and Schindler, since it would contradict the claim that Tr-E is sufficient for reasoning about embedded truth-ascriptions. ${ }^{12}$ It would seem, then, that Picollo and Schindler can maintain that Tr-E suffices to account for cases of embedded agreement and disagreement only if $\mathrm{Tr}-\mathrm{I}$ is already assumed to hold. ${ }^{13}$

In conclusion, Field's argument overshoots because transparency is not necessary for the expressive role of truth, even in cases of embedded truth-ascriptions, while Picollo and Schindler's argument undershoots because Tr-E alone is not sufficient. We would rather suggest that in medio stat virtus: namely, the combination of both Tr-E and Tr-I is exactly what is required by the expressive role of 'true'. To be sure, while this shows that the Argument for Transparency misses its target, our conclusion seemingly vindicates a version of the Argument for Naïveté-one according to which the expressive role of 'true' requires full Tr-E and Tr-I and, for this reason, is incompatible with classical theories of truth. As a result, it would seem, the classical theorist is still left with an uncomfortable choice between classical logic, on the one hand, and the expressive role of 'true', on the other. In the next section, however, we argue that this is a false dilemma. There exist contextualist versions of Tr-E and Tr-I that fully underwrite the expressive role of 'true' and that yet do not require one to adopt a non-classical theory of truth.

\subsection{Contextualist Agreement and Disagreement}

The problem, for most classical theories, is that they cannot validate both Tr-E and Tr-I. As the Knower Paradox shows (Kaplan and Montague, 1960; Myhill, 1960), they cannot simultaneously validate principles of truth introduction and truth elimination, even if one of the two is severely restricted (i.e. restricted to sentences that are proven from no assumptions). While non-hierarchical classical theories simply give up one between Tr-E and Tr-I, hierarchical theories typically still validate a certain version of Tr-I. In particular, contextualist theories feature versions of both Tr-E and Tr-I, where, crucially, the introduction rule is context-shifting.

Our plan is as follows. We first introduce orthodox contextualist approaches and point to some of their limitations (\$5.2.1). We then sketch our own preferred contextualist approach (\$5.2.2) and argue that it is expressively adequate, in the sense of validating both 
unembedded and embedded cases of agreement and disagreement (§5.2.3). We finally close by considering some objection and by offering some replies on the contextualist's behalf (§5.2.4).

\subsubsection{An Overview of Orthodox Truth-Theoretic Contextualism}

Contextualist proposals (first advanced by Charles Parsons, 1974 and extensively developed by Michael Glanzberg, 2001, 2004a, 2015) offer a more sophisticated, and nuanced, interpretation of truth-theoretical statements. Contextualists typically assume that propositions are the primary bearers of truth and falsity. Accordingly, a sentence $\varphi$ is true in a context $\alpha$ if and only if it expresses a true proposition in $\alpha$ (Kaplan, 1989, p. 522). Following Glanzberg (2001, 2004a), we formalise the right-hand side of this biconditional as follows:

$$
\exists_{\alpha} p(\operatorname{Exp}(\ulcorner\varphi\urcorner, p, \alpha) \wedge \operatorname{Tr}(p)),
$$

where $\operatorname{Exp}(\ulcorner\varphi\urcorner, p, \alpha)$ reads ' $\varphi$ expresses $p$ in $\alpha$ ', $\exists_{\alpha} p$ expresses existential quantification over a domain of propositions determined by the context $\alpha$, and $\operatorname{Tr}$ expresses propositional truth. To further simplify notation, we write $\operatorname{Tr}_{\alpha}(\ulcorner\varphi\urcorner)$ for $\exists_{\alpha} p(\operatorname{Exp}(\ulcorner\varphi\urcorner, p, \alpha) \wedge \operatorname{Tr}(p))$ (operating under the assumption that, in each given context, every sentence expresses at most one proposition). Still following Glanzberg, we assume that any adequate (naïve) contextualist theory contains all the instances of the following schema:

$$
\text { (CTS) } \forall_{\alpha} p[\operatorname{Exp}(\ulcorner\varphi\urcorner, p, \alpha) \rightarrow(\varphi \leftrightarrow \operatorname{Tr}(p))] .
$$

In contextualist approaches, a Liar sentence $\lambda$ is interpreted as the claim that $\lambda$ doesn't express a true proposition in a given context $\alpha$. In these theories, $\lambda$ doesn't express a true proposition in $\alpha$, but expresses a true proposition in a new, richer context $\beta$. On the further assumption that $\lambda$, thus understood, involves propositional quantifiers whose domains vary with context, the Liar reasoning is blocked. Since the Liar reasoning is now interpreted as showing that $\lambda$ is not true in $\alpha$ but true in $\beta$, it is no longer paradoxical and its conclusion no longer contradictory.

Here is, in more detail, the contextualist construal of the Liar reasoning (we informally follow Glanzberg, 2004a, pp. 33-34). Let $\gamma$ be the initial context of reasoning and let $\lambda$ be a sentence equivalent to ' $\lambda$ doesn't express a true proposition in $\gamma^{\prime}$ :

$$
\lambda \leftrightarrow \neg \exists_{\gamma} p(\operatorname{Exp}(\ulcorner\lambda\urcorner, p, \gamma) \wedge \operatorname{Tr}(p))
$$

(the latter can also be written as $\neg \operatorname{Tr}_{\gamma}(\ulcorner\lambda\urcorner)$ ).

One assumes that $\lambda$ expresses a proposition in $\gamma$, and reasons (using CTS) that any such proposition $p$ is true if and only if it is not true-a 
contradiction. Hence, one must negate and discharge the initial supposition that $\lambda$ expresses a proposition, and conclude that

$$
\lambda \text { does not express a proposition in } \gamma \text {. }
$$

One can then reason as follows:

(18) But, then, $\lambda$ does not express a true proposition in $\gamma \quad[17, \operatorname{logic}]$

(19) Then, $\lambda$

[Definition of $\lambda$ ]

(20) Thus, $\lambda$ expresses a true proposition in $\gamma$

$[19, \operatorname{Tr}-1]$

Clearly, (18) and (20) contradict each other.

Contextualists maintain that the foregoing argument is not valid. ${ }^{14}$ In particular, a context shift takes place between (18) and (20)-more specifically between (19) and (20) (Murzi and Rossi, 2018)—so that (20) should be interpreted as:

$\left(20^{\star}\right) \lambda$ expresses a true proposition in $\gamma^{\prime}$ (for a context $\gamma^{\prime}$ different from $\gamma$ ).

Since (18) and $\left(20^{\star}\right)$ are consistent, the Liar reasoning is blocked. ${ }^{15}$

Contextualist theories have several advantages. To name but a few: they retain classical logic; they model paradoxical reasonings as intuitively correct arguments, making them consistent by uncovering a context-shift in them; finally, they improve on some of our best theories of truth. For example, contextualist theories à la Glanzberg validate the truth-theoretical axioms of KF + Cons-that is, Feferman's classical axiomatization (Feferman, 1991) of Kripke's theory of truth (Kripke, 1975), plus the consistency axiom, ensuring that no sentence is both true and false. ${ }^{16}$ However, while $\mathrm{KF}+$ Cons is incompatible with Tr-In, its contextualist version can be consistently closed under a context-shifting version of Tr-In, while retaining the virtues of the original theory.

On the other hand, on the orthodox contextualist view, quantification is always restricted to less than absolutely comprehensive domains. The reason, once again, is semantic paradox. In order to consistently interpret the Liar reasoning, $\lambda$ is interpreted twice: first, in the initial context $\gamma$ in which it does not express a true proposition, then in $\gamma^{\prime}$ where it does (see (18) and $\left(20^{\star}\right)$ above). More specifically, the truthintroduction rule triggers a shift in the domain for the propositional quantifier-from a starting domain $\alpha$ to a domain $\beta$, where the propositions available for expressions in $\beta$ strictly include those of $\alpha .{ }^{17}$ And since one can run paradoxical reasonings in any given context, and paradoxical reasonings extend-on the contextualist construal-any given domain of quantification, it seemingly follows that quantifiers can 
never range over absolutely everything. However, this feature of the approach clearly overshoots. Consider the following sentence:

\section{(21) Everything is self-identical.}

On an orthodox contextualist construal, even in sentences such as (21), the quantifiers range over less than absolutely everything. But this is unacceptable. Intuitively, the quantifier in (21) ranges over absolutely everything that there is, and a restricted reading of (21) would simply misconstrue it. ${ }^{18}$

In order to overcome this problem, we adopt the bicontextualist theory developed in Rossi (2021). The theory provides an absolutely general interpretation of unproblematic sentences such as (21), while preserving the context-shifting interpretation of paradoxical sentences and the positive aspects of orthodox contextualism à la Glanzberg more generally. ${ }^{19}$

\subsubsection{Bicontextualism}

The basic bicontextualist idea is that whether quantification can be absolutely general depends on the sentence in which the quantifiers appear: in some sentences (call them 'unparadoxical'), quantifiers can be interpreted as absolutely unrestricted; in others (call them 'paradoxical'), they cannot. Having distinguished between unparadoxical and paradoxical sentences, bicontextualist semantics provides an absolutist semantics for the former and a relativist semantics for the latter. In a slogan, bicontextualist semantics recaptures absolute generality whenever possible, just like standard non-classical approaches recapture classical logic whenever possible. ${ }^{20}$

In a bicontextualist semantics, we model context shift by moving from an interpretation for a given language to an interpretation for another, more expressive language. For this reason, we employ a proper class of first-order languages

$$
\mathfrak{L}:=\mathcal{L}_{0}, \mathcal{L}_{1}, \ldots, \mathcal{L}_{\alpha}, \ldots
$$

where every $\mathcal{L}_{\alpha} \in \mathfrak{L}$ is expressive enough to admit the encoding of syntactic notions and $\cup_{\alpha<\beta} \mathcal{L}_{\alpha} \subseteq \mathcal{L}_{\beta}$, for $\alpha<\beta$. We write $\operatorname{Tr}_{\alpha}(\ulcorner\varphi\urcorner)$ for ' $\varphi$ is true in $\alpha$ '. For simplicity, we take sentences as truth-bearers: as pointed out by Parsons (1974, p. 391 and following), this reformulation is harmless, and the talk about propositions can always be recovered by replacing ${ }^{\prime}\ulcorner\varphi\urcorner$ is true in $\alpha$ ' with ' $\ulcorner\varphi\urcorner$ expresses a true proposition in $\alpha$ '.

\section{Absolutist Semantics}

In order to construct second-order interpretations for unrestricted firstorder languages, we follow Rayo and Uzquiano (1999) and Rayo and 
Williamson (2003), adapting their approach to the case in which such languages contain a self-applicable truth predicate. We informally work in second-order Zermelo-Frankel set theory (ZFC2). First, we define a predicate ' $X$ is a model' in $\mathcal{L}_{\mathrm{ZFC} 2}, \mathbb{M}(X)$. We skip the (lengthy) definition of $\mathbb{M}(X)$, but it can be paraphrased as follows: there is at least one $x$ s.t. $X x$ holds (models are non-empty), and $X x$ holds whenever $x$ specifies a domain, or the denotation of an individual constant, or the extension of a relation. The notions of 'domain', 'denotation', and 'extension' are given second-order paraphrases in the official definition of $\mathbb{M}(X)$.

We similarly define in $\mathcal{L}_{\mathrm{ZFC} 2}$ a second-order predicate $\mathbb{A}(s, X)$, for ' $s$ is a variable assignment relative to $X$ ', and $\mathbb{V}\left(s, t, v_{i}, X\right)$ for ' $t$ is a $v_{i}$-variant of $s$ relative to $X$ '. The three second-order predicates $\mathbb{M}(X), \mathbb{A}(s, X)$, and $\mathbb{V}\left(s, t, v_{i}, X\right)$ are now used to define in $\mathcal{L}_{\mathrm{ZFC} 2}$ an absolutist notion of satisfaction the object-languages in $\mathfrak{L}$, patterned after the construction of the least fixed point of Kripke's theory of truth (1975).

Let $x$ be a first-order variable, and $X, Y$, and $Z$ be second-order variables. $\operatorname{KSat}_{\alpha}(x, X, Y, Z)$ holds just in case $x$ is (the code of) a sentence that is satisfied in the (second-order) model $X$ whose (second-order analogue of) domain is $Y$, relative to the accepted $Z$ (note that, for simplicity reasons, the definition combines the second-order notions of model and variable assignment into one). The variable $Z$ stands for the (codes of) sentences that are assumed to be true in the construction of the Kripkean model. We skip the (lenghty definition) of KSat for space reasons-the interested reader can consult Rossi (2021). ${ }^{21}$

We can now use KSat to define the set of absolutely general truths of each language $\mathcal{L}_{\alpha}$.

Definition 5.5.2. For every $\mathcal{L}_{\alpha} \in \mathfrak{Q}$, the set of absolutely general truths of $\mathcal{L}_{\alpha}$ is defined as follows:

$$
\operatorname{Abs}_{\alpha}:=\left\{\varphi \in \mathcal{L}_{\alpha} \mid \operatorname{KSat}_{\alpha}(\varphi, X, Y, \varnothing)\right\} .
$$

$\mathrm{Abs}_{\alpha}$ contains the sentences that are in the least fixed point for the language $\mathcal{L}_{\alpha}$ whose quantifiers are interpreted as ranging over the domain encoded by $X$, possibly absolutely everything.

\section{Relativist Semantics}

In order to obtain a relativist semantics for paradoxical sentences, we adapt Glanzberg's original treatment to the present setting. In Glanzberg's approach, different (set-sized) 'closed-off' Kripkean fixed points are used to interpret paradoxical sentences in different contexts (the notion of 'closing-off' is sketched in what follows). However, in a bicontextualist semantics, the relativist interpretation has to apply only 
to the paradoxical sentences. Therefore, the paradoxical sentences of $\mathcal{L}_{\alpha}$ given by the closing off of a fixed point, minus that fixed point.

We now outline how to construct a succession of Kripkean closed-off fixed points, and then explicitly define the relatively general truths, for each $\mathcal{L}_{\alpha}$. Let $\varnothing$ be a predicate with an empty extension, $\mathcal{M}_{\alpha}$ be a model of the non-semantic fragment of $\mathcal{L}_{\alpha}$, and $\mathrm{M}_{\alpha}$ be its support. The relativistic closing off for $\mathcal{L}_{0}$ is:

$$
\text { C-Off }{ }_{0}:=\left\{\varphi \in \operatorname{Sent}_{\mathcal{L}_{0}} \mid\left\langle\mathcal{M}_{0},\left\{\psi \in \mathcal{L}_{0} \mid \mathrm{KSat}_{0}\left(\ulcorner\varphi\urcorner, \mathcal{M}_{0}, \mathrm{M}_{0}, \varnothing\right)\right\}\right\rangle \models \varphi\right\} .
$$

For $\alpha>0$, the closing-off of $\mathcal{L}_{\alpha}$ is:

$$
\begin{aligned}
& \mathrm{C}_{-} \mathrm{Off}_{\alpha}:=\left\{\varphi \in \operatorname{Sent}_{\mathcal{L}_{\alpha}} \mid\right. \\
&\left.\left\langle\mathcal{M}_{\alpha},\left\{\psi \in \mathcal{L}_{\alpha} \mid \mathrm{KSat}_{\alpha}\left(\ulcorner\psi\urcorner, \mathcal{M}_{\alpha}, \mathrm{M}_{\alpha} \cup \bigcup_{\beta<\alpha} \mathrm{C}-\mathrm{Off}_{\beta}\right), \bigcup_{\beta<\alpha} \mathrm{C}-\mathrm{Off}_{\beta}\right)\right\rangle \models \varphi\right\} .
\end{aligned}
$$

This succession of closed-off fixed points treats paradoxical sentences exactly as in Glanzberg's original approach. Consider a Liar in $\mathcal{L}_{0}$, call it $\lambda_{0} . \lambda_{0}$ is not in the extension of the fixed point defined by $\mathrm{KSat}_{0}\left(\ulcorner\varphi\urcorner, \mathcal{M}_{0}, \mathrm{M}_{0}, \varnothing\right)$. Since $\mathrm{KSat}_{0}\left(\ulcorner\varphi\urcorner, \mathcal{M}_{0}, \mathrm{M}_{0}, \varnothing\right)$ yields the extension of $\operatorname{Tr}_{0}, \lambda_{0}$ is not in it. Therefore:

$$
\begin{aligned}
\left\langle\mathcal{M}_{0},\left\{\psi \in \mathcal{L}_{0} \mid \operatorname{KSat}_{0}\left(\ulcorner\varphi\urcorner, \mathcal{M}_{0}, \mathrm{M}_{0}, \varnothing\right)\right\}\right\rangle & \not \operatorname{Tr}_{0}\left(\left\ulcorner\lambda_{0}\right\urcorner\right) \\
& \models \neg \operatorname{Tr}_{0}\left(\left\ulcorner\lambda_{0}\right\urcorner\right) \\
& \models \lambda_{0}
\end{aligned}
$$

However, since $\lambda_{0} \in \mathrm{C}$-Off ${ }_{0}$, when one builds a fixed point for $\mathcal{L}_{1}$ over C-Off ${ }_{0}, \lambda_{0}$ goes into the extension of $\mathrm{Tr}_{1}$. Therefore:

$$
\left\langle\mathcal{M}_{1}, \operatorname{KSat}_{1}\left(\ulcorner\varphi\urcorner, \mathcal{M}_{1}, \mathrm{M}_{1} \cup \mathrm{C}-\mathrm{Off}_{0}, \mathrm{C}-\mathrm{Off}_{0}\right)\right\rangle \models \operatorname{Tr}_{1}\left(\left\ulcorner\lambda_{0}\right\urcorner\right) .
$$

That is, $\lambda_{0}$ does not express a true proposition of $\mathcal{L}_{0}$, but it expresses a true proposition of $\mathcal{L}_{1}$, as sketched in the reasoning $(18)-\left(20^{\star}\right)$. Other paradoxical sentences are treated in the very same way.

Now that we have defined the closed-off fixed points, we can easily define the paradoxical sentences.

Definition 5.2.3. For every $\mathcal{L}_{\alpha} \in \mathfrak{Q}$, the set of relatively general truths of $\mathcal{L}_{\alpha}$, in symbols $\mathrm{Rel}_{\alpha}$, is defined as the set of sentences in C-Off ${ }_{\alpha}$, minus the sentences in $\mathrm{Abs}_{\alpha}$ and the sentences $\varphi$ s.t. $\varphi \in \mathrm{C}$-Off ${ }_{\alpha}$ but $\neg \varphi \in \mathrm{Abs}_{\alpha}$ or vice versa.

The relatively general truths of $\mathcal{L}_{\alpha}$ are exactly the truths of $\mathcal{L}_{\alpha}$ 's closed-off fixed point minus $\mathcal{L}_{\alpha}$ 's absolutely general truths. 


\section{Bicontextualism in Full}

We can now finally define a proper bicontextualist theory of truth.

Definition 5.2.4. For every $\mathcal{L}_{\alpha} \in \mathfrak{L}$, and every $\{\Gamma, \varphi\} \subseteq \mathcal{L}_{\alpha}$, the argument from $\Gamma$ to $\varphi$ is bicontextually valid, in symbols $\Gamma \models_{\alpha}^{\text {bc }} \varphi$, if and only if:

all the sentences in $\Gamma$ are in $\mathrm{Abs}_{\alpha} \cup \operatorname{Rel}_{\alpha}$, so is $\varphi$.

For every $\mathcal{L}_{\alpha} \in \mathfrak{L}$, the bicontextualist semantics for $\mathcal{L}_{\alpha}$ interprets all the unparadoxical sentences over a possibly absolutely unrestricted domain, and the paradoxical ones over a restricted, set-sized domain.

Bicontextualism delivers a strong theory of truth. Among other things, it validates all the axioms of $\mathrm{KF}+$ Cons for every language $\mathcal{L}_{\alpha}$. More importantly for our present purposes, however, it validates forms of truth-introduction and elimination which can be used to recover talk about Agreement and Disagreement, to which we now turn.

\subsubsection{Bicontextualism Agreement and Disagreement}

Both orthodox contextualist semantics and bicontextualism validate the following introduction rule for the the truth predicate (we formulate the rules as inferences from sequents to sequents to make fully clear the context in which each sentence occurs):

$$
\text { (C-Tr-I) If } \Gamma \models_{\alpha}^{\mathrm{bc}} \varphi \text {, then } \Gamma \models_{\beta}^{\mathrm{bc}} \operatorname{Tr}_{\beta}(\ulcorner\varphi\urcorner) \text {. }
$$

Its contrapositive governs the elimination of the negated truth predicate:

$$
\text { (C-ᄀTr-E) If } \Gamma \models_{\beta}^{\mathrm{bc}} \neg \operatorname{Tr}_{\beta}(\ulcorner\varphi\urcorner) \text {, then } \Gamma \models_{\alpha}^{\mathrm{bc}} \neg \varphi \text {, }
$$

where $\beta$ is strictly greater than $\alpha$. While the latter two rules may require a shift of context, the corresponding converse rules are not contextshifting:

$$
\begin{aligned}
& \text { (C-Tr-E) If } \Gamma \models_{\alpha}^{\mathrm{bc}} \operatorname{Tr}_{\alpha}(\ulcorner\varphi\urcorner) \text {, then } \Gamma \models_{\alpha}^{\mathrm{bc}} \varphi \text {; } \\
& \text { (C-ᄀTr-I) If } \Gamma \models_{\alpha}^{\mathrm{bc}} \neg \varphi \text {, then } \Gamma \models_{\alpha}^{\mathrm{bc}} \neg \operatorname{Tr}_{\alpha}(\ulcorner\varphi\urcorner) \text {. }
\end{aligned}
$$

As we have seen in $§ 5.1$, naïve principles such as Tr-E and Tr-I seem to be required in order to model speeches such as Agreement and DisagreeMENT. However, Agreement and Disagreement can be equally modelled by means of a contextualist truth predicate. In particular, speeches requiring a truth introduction principle can be modelled using the contextualist rules C-Tr-I and C-ᄀTr-E. For instance, contextualists may 
interpret DisAGREEMENT as follows (letting ' $\varsigma(x, y)$ ' formalise the claim ' $x$ said $\left.y^{\prime}\right)$ :

$$
\frac{\frac{\forall x\left[\varsigma(l, x) \rightarrow \neg \operatorname{Tr}_{\beta}(x)\right]}{\varsigma(l,\ulcorner\varphi\urcorner) \rightarrow \neg \operatorname{Tr}_{\beta}(\ulcorner\varphi\urcorner)} \forall-\mathrm{E} \quad \varsigma(l,\ulcorner\varphi\urcorner)}{\frac{\neg \operatorname{Tr}_{\beta}(\ulcorner\varphi\urcorner)}{\neg \varphi} \text { C- --Tr-E }} \rightarrow \text {-E }
$$

where the first three lines occur in $\beta$, the fourth line occurs in $\alpha$, and $\beta$ is strictly greater than $\alpha$.

Contextualist approaches, then, can adequately model key uses of the truth predicate such as AgreEment and DisagreEMENT, modulo the possible occurrence of context shifts. In addition-as shown in \$5.1.2-cases of embedded agreement and disagreement can also be modelled by means of contextualist principles of truth introduction and elimination. However, orthodox contextualist theories and the bicontextualist theory we favour differ in one important respect. While the former theories always interpret C- $\neg$ Tr-I and C- $\neg$ Tr-E as context-shifting, in a bicontextualist framework these rules are context-shifting only when applied to paradoxical sentences. By contrast, non-paradoxical sentences do not trigger context-shifts and are interpreted by the absolutist fragment of the semantics. Therefore, cases of (embedded or non-embedded) agreement and disagreement involving non-paradoxical sentences are modelled as if the truth predicate were naïve, i.e. by means of perfectly symmetrical introduction and elimination rules.

Let us now consider again the classical rejoinder given in \$5.1.1. There, we argued that the classical truth-theorist might remain unconvinced by the intuitive validity of schematic arguments such as AGREEMENT and DisAgREEMENT because they seem to have instances-e.g. those involving paradoxical sentences-that are not clearly compelling. Bicontextualist semantics seems to do justice to this intuition: instances of Agreement and Disagreement that do not involve paradoxical sentences can be modelled naïvely (as in most classical approaches). In addition, however, instances involving paradoxical sentences can also be modelled. However, in this case the introduction of the truth predicate and the elimination of the negated truth predicate semantically correspond to the articulation of a truth-theory for the sentences of a given language, and therefore require a stronger context in which they can be given.

We now turn to some potential objections-in particular, objections concerning whether it is always possible to model agreement and disagreement in a bipartite and hierarchical manner. ${ }^{22}$ 


\subsubsection{Objections and Replies}

We consider three different kinds of objections: objections from semantic blindness, Dean-Nixon cases, and objections from ineffability, to the effect that systematic domain restrictions cannot be coherently stated.

\section{Semantic Blindness}

Consider a blind ascription, such as

(6) Everything Lois said yesterday is not true,

and suppose we don't know what Lois said yesterday. Kripke (1975, p. 695 and ff.) famously argued, against Tarskian hierarchical approaches, that one may not be in a position to assign a 'level' to the truth predicate occurring in (6). ${ }^{23}$ In a contextualist framework, though, Kripke's point is not well taken. The objection assumes that, in order to successfully use sentences such as (6), a speaker must know the interpretation of 'true' in a given context. In our framework, (6) is interpreted as

$\left(6^{\star}\right)$ Everything Lois said yesterday expresses a true proposition in some context $\alpha$.

More formally:

(6 $\left.6^{\star \star}\right) \forall x\left(\varsigma(\right.$ Lois, $\left.x) \rightarrow \operatorname{Tr}_{\alpha}(\ulcorner\varphi\urcorner)\right)$.

Thus, in our framework, the objection postulates that a speaker must know in which context the truth ascription in $\left(6^{\star \star}\right)$ must be interpreted. However, in general speakers need not know the features of context that are relevant for the interpretation of context-sensitive expressions. Consider the sentence

(22) It's cloudy now.

Speakers utter sentences like these even if they don't exactly know what time it is. Granted, in order to know what (22) says, speakers must first find out what time it is. But a speaker doesn't need to know the time in order to successfully use sentences like (16). Similarly, even if we don't know what Lois said yesterday, we are still able to use (6) to successfully attribute truth or untruth to what she says, even though we are not always in a position to know the content of what we have just agreed or disagreed on. All is required from a contextualist semantics for 'true' is that there be a context that provides a suitable interpretation of the context-sensitive 
expressions in (6). And contextualist approaches standardly satisfy this requirement.

\section{Nixon-Dean Cases}

In less liberal hierarchical approaches, such as Tarski's, certain everyday speeches cannot be coherently interpreted. Suppose Trump and Cohen only utter, respectively, the following sentences (cf. Kripke, 1975, pp. 695-696):

(23) Everything Cohen says about the hush payment is true;

(24) Everything Trump says about the hush payment is not true.

In a standard Tarskian framework, (23) and (24) cannot be interpreted, since each sentence would need to involve a truth predicate of higher level than that of the truth predicate occurring in the other sentencewhich is of course impossible. However, as Glanzberg (2015, p. 233) points out, the objection doesn't apply to more liberal hierarchical theories. In particular, contextualist theories à la Glanzberg use iterations of Kripkean fixed points to interpret paradoxical sentences (and only those). Therefore, (23) and (24) can be interpreted exactly as in Kripke's theory (and closed-off versions of the theory) in every such fixed point. ${ }^{24}$

It might be insisted that if the speakers explicitly talk about the context they're in, a version of Kripke's objection can still be made to work (Field, 2008, pp. 217-218). For suppose Trump and Cohen only utter, respectively, in contexts $\delta$ and $\gamma$ :

$\left(23^{\star}\right)$ Everything Cohen says in context $\gamma$ about the hush payment is true;

$\left(24^{\star}\right)$ Everything Trump says in context $\delta$ about the hush payment is not true.

Then, as Field (2008) put it,

for one guy to succeed in saying what he intended he must pick a strictly higher subscript than the other. Because of this, there is pressure for [Trump] and [Cohen] to get into a subscript contest.

However, our treatment of $\left(23^{\star}\right)$ and $\left(24^{\star}\right)$ generalises to (23) and (24). The latter two utterances can be given a standard contextualist treatment, interpreting them as a simple variant of the Liar reasoning. More precisely, since both sentences are paradoxical, they are interpreted by some Kripke fixed point in which neither of them expresses 
a true proposition. Such Kripke fixed points corresponds to interpreting $\left(23^{\star}\right)$ and $\left(24^{\star}\right)$ in some suitable context $\alpha$ that extends the contexts over which both $\left(23^{\star}\right)$ and $\left(24^{\star}\right)$ quantify. As with the original Liar sentence $\lambda$, both $\left(23^{\star}\right)$ and $\left(24^{\star}\right)$ can then be shown to express propositions in a context $\beta$ that extends $\alpha$.

\section{Stating the View}

Field (2008, pp. 220-221) considers a further objection, to the effect that contextualist theories cannot coherently interpret a theorist's disagreement with the contextualist theory as a whole. For suppose one wishes to assert that the contextualist theory is not true. Then, it might seem that one needs to quantify over all the propositions that are expressed in all the contexts, which is something that contextualist theories cannot do (with the intended absolutely unrestricted interpretation). This is an instance of a more general objection, to the effect that hierarchical approaches to the paradoxes lack the expressive resources to talk about the whole hierarchy (in our case: the whole hierarchy of propositions in context). ${ }^{25}$ We now provide a quick sketch of how this objection can be addressed in a contextualist framework.

Bicontextualists place some limits on absolutely unrestricted quantification: the quantifiers of paradoxical sentences are necessarily restricted to less than absolutely general domains. Yet, in many contexts, they are able to quantify over absolutely everything. How to characterise, more precisely, such a view? This is an instance of a more general problem afflicting the two main views about generality: absolutism, according to which it is possible to quantify over absolutely everything, and relativism, according to which quantification is never absolutely general. On one hand, relativists face the objection that they cannot express their own view, if it is expressed as the thought that we cannot quantify over absolutely everything. After all, the objection goes, this very thought presupposes absolute generality. ${ }^{26}$ On the other, absolutists are accused of only being able to offer essentially meta-theoretic and hence, one might argue, unintelligible statements of their view. What is the status of bicontextualism in this landscape? The answer, in a nutshell, is that bicontextualism is essentially an absolutist view, with some restrictions. But it is neither incoherent nor unintelligible.

The meta-theory of bicontextualism is absolutist, i.e. it has the resources to express absolutely general quantification. More specifically, some open formulae are satisfied by everything, i.e. by a collection of things whose size exceeds that of any set, however large. Accordingly, the bicontextualist can express her view as a conjunction of two claims: 
(25) There are open formulae that are satisfied by absolutely everything;

(26) There are open formulae that are only satisfied by set-many things.

Here 'absolutely everything' is shorthand for the satisfaction clause for universally quantified sentences, formulated in a higher-order language. Hence, both (25) and (26) are essentially meta-linguistic claims.

It might be objected that this is deeply unsatisfactory, on the grounds that (25) and (26) look like perfectly ordinary object-language sentences that ought to be expressed in the object-language. Indeed, it might be insisted that English is not divided into English and meta-English and that, in principle, everything that is expressible in the meta-language ought to also be expressible in the object-language-including (25) and (26). After all, what is the point of having a type-free truth predicate, if not to formulate the interpretation (i.e. a traditionally meta-theoretical notion) for a theory $S$ in $S$ ?

One first point to notice here is that (25) and (26)'s meta-linguistic character isn't specific to bicontextualism. For instance, (25) could be just as well taken to express the claim that it is possible to quantify over absolutely everything-a standard statement of the absolutist view (Williamson, 2003). If stating one's view about the interpretation of quantifiers in the meta-language is problematic, then it is problematic for the absolutist and the relativist just as well.

More importantly, the objection overshoots: it is false in general that everything that is the meta-language ought to be expressible in the object-language; only what is already expressible in English should. And, in this respect, there is an asymmetry between truth and higherorder quantification. The reason why a type-free truth predicate ought to be part of the object-language is the simple existence of English speeches that make an essential use of it-speeches such as AGreEMENT, DisAGREEMENT, and the like. However, no parallel motivation for expressing higher-order quantification in the object-language is available. To see this, notice that a genuine higher-order quantification amounts to quantifying into predicate position, i.e. quantifying over something that also figures as a predicate in the expression that follows the quantifier. For instance, a faithful reading of an expression of the form $\forall X \forall x(X x)$ would amount to something like 'Every $X X$ s every $x$ '. That is, $X$ would need to simultaneously be the syntactic object to which the quantifier applies and the predicate in the expression following the quantifier. Yet this doesn't seem to be possible in languages such as English. To be sure, plenty of English paraphrases of $\forall X \forall x(X x)$ are available. For instance, 'For every predicate, everything satisfies it'. These translations 
are systematically misleading, though: they effectively treat $X$ as a firstorder variable, because, in the paraphrases, $X$ does not serve as a predicate in the expression following the quantifier.

\subsection{Concluding Remarks}

It is usually thought that Tarski's Theorem forces on us an uncomfortable dilemma: either adopt a non-classical theory of truth, or restrict some of the naïve truth principles and 'seriously cripple our ability to make generalizations' (Field, 2008, p. 349). In turn, this has led many to adopt a non-classical theory of truth. However, there are good reasons for thinking that non-classical approaches are fundamentally misguided (Murzi and Rossi, 2020): they inevitably give rise to revenge paradoxes they are either unable to block, or they can only block at unacceptable costs. We hope to have shown that this is a false dilemma. The expressive role of truth doesn't require naïveté: contextualist principles of truth (and untruth) introduction and elimination suffice. There are several possible reasons for adopting a non-classical theory of truth. But the legitimate desire to preserve, on the face of paradox, our ability to make generalisations is not one of them.

\section{Acknowledgements}

We are grateful to Carlo Nicolai and Johannes Stern for very helpful comments on a previous draft of this paper, as well as to the FWF (Stand-Alone Grant P29716-G24), for generous financial support during the time this article was written.

\section{Notes}

1. Even if the truth-predicate is naïve, certain instances of the naïve principles for truth can plausibly fail in opaque contexts, quite independently of the paradoxes. For instance, 'Anne believes that every even number greater than two is the sum of two primes' may not imply 'Anne believes that "every even number greater than two is the sum of two primes" is true', for the simple reason that Anne may lack the concept of truth, or that she may systematically refuse to apply it to arithmetical sentences.

2. Typically, we leave the formal system unspecified and assume that the arguments we model are formalised in a theory featuring logical rules, a modicum of syntax theory (in order to have a well-behaved name-forming device $\ulcorner$.$\urcorner ),$ and semantic rules (governing the truth predicate).

3. Even if they don't contain all instances of Tr-I and Tr-E, classical theories allow applications of Tr-I and Tr-E to (more or less large) classes of sentences. These typically include non-semantic sentences, or sentences involving iterated applications of the truth predicate to non-semantic sentences. For instance, even classical typed theories of truth allow instances of Tr-I and Tr-E for sentences not containing the truth predicate (see for example the theories TB and 
UTB Halbach, 2011, Ch. 7), while strong classical theories allow instances of the naïve principles for possibly very complex iterated applications of the truth predicate to non-semantic sentences (see for example Feferman, 1991, 2008).

4. Note that the foregoing considerations also apply to instances of AGREEMENT and DisagreEment involving obviously false sentences, such as 'Donald Trump is a theorem of Peano Arithmetic'.

5. Classical theorists typically restrict naïve principles to non-paradoxical sentences, for some suitable understanding of the notion of paradoxicality. Similarly, non-classical theorists restrict classical principles to non-paradoxical sentences. For a discussion of whether this in turn leads to certain revenge problems, see Bacon (2015), for the classical case, and Murzi and Rossi (2020), for the non-classical one.

6. We consider a scenario in which one wishes to disagree with everything that was written in the report. The case of an embedded disagreement involving an antecedent of the form 'something in the Conyers report is untrue' is dealt with similarly.

7. The line labelled 'CR' stands for the rule of classical reductio. Also, we use the assumption $C(\ulcorner v\urcorner)$, rather than $C(\ulcorner\chi\urcorner)$, for simplicity; however, if $v$ is the disjunction of the claims in the Conyers report and $\chi$ is their conjunction, then the former clearly follows from the latter, so we could have equally assumed $\mathrm{C}(\ulcorner\chi\urcorner)$.

8. We use the index $(i \times k)$ to make sure that it is sufficiently high not to clash with the indices of the open assumptions $\varphi_{1}$, which are to be closed and discarded later.

9. There might be exceptions in some substructural settings.

10. It should be noted that Picollo and Schindler do not endorse the claim that results such as the one above show that truth-theoretical generalisations 'express' all their instances.

11. It should be noted that a (sufficiently expressive) classical base theory $S$ that features $\forall x\left(\mathrm{C}(x) \leftrightarrow x=\left\ulcorner\varphi_{1}\right\urcorner \vee \ldots \vee x=\left\ulcorner\varphi_{n}\right\urcorner\right)$ amongst its axioms can be consistently closed under all the instances of the inference from $\forall x(\mathrm{C}(x) \rightarrow \operatorname{Tr}(x)) \rightarrow \psi$ to $\varphi_{1} \wedge \ldots \wedge \varphi_{n} \rightarrow \psi$. To see that the first inference goes through it is sufficient to consider any model of the form $\mathcal{A}:=\langle\mathcal{M}, A\rangle$, where $\mathcal{M}$ is a model of the truth-free part of the language of $S$ and $A$ is the set $\left\{x \in M \mid x=\left\ulcorner\varphi_{1}\right\urcorner\right.$ or $\ldots$ or $\left.x=\left\ulcorner\varphi_{n}\right\urcorner\right\}$ which serves as the extension of $\operatorname{Tr}$ in $M$ (the support of $\mathcal{M}$ ). The converse inference, i.e. from $\varphi_{1} \wedge \ldots \wedge \varphi_{n} \rightarrow \psi$ to $\forall x(\mathrm{C}(x) \rightarrow \operatorname{Tr}(x)) \rightarrow \psi$, impossibly requires closure under unrestricted Tr-E.

12. We are indebted to Carlo Nicolai for bringing this point to our attention.

13. Picollo and Schindler (2019) further discuss the expressive role of 'true'. In this more recent paper, they too argue that both Tr-I and Tr-E are essential for the expressive role of 'true'.

14. See e.g. Parsons (1974) and Glanzberg (2001, 2004a).

15. On why exactly context shifts in the course of the Liar reasoning, see Glanzberg (2004b, 2015), Gauker (2006), and Murzi and Rossi (2018).

16. For more details on KF and its extensions, see Field (2008, Chapters 7 and 13) and Halbach (2011, Chapter 15).

17. To be sure, the question arises why truth-introduction principles induce a context shift. For instance, Glanzberg (2004b, 2015) argues that when we explicitly articulate our acceptance of a given theory $S$, we are committed to principles that determine the truth-conditions of $S$ 's sentences, that is, we are committed to a suitable theory of truth $S^{\prime}$ for $S$. Since $S^{\prime}$ 's truth predicate cannot be defined in the theory $S$ itself, on pain 


\section{Julien Murzi and Lorenzo Rossi}

of triviality, a more expressive theory is required, whence the context-shifting properties of truth-theoretical reflection (see also Murzi and Rossi, 2018).

18. Williamson (2003) further argues that absolutely general quantification is crucial for philosophical and scientific theorizing and that, for this reason, any view on which quantification is necessarily restricted is at odds with the level of generality required by scientific reasoning.

19. For reasons of space, we only offer a brief, informal outline of the theory. The following subsection draws extensively from the more detailed presentation in Rossi (2021). We also assume some familiarity with the models constructed in Kripke (1975). The reader uninterested in the outline of the theory can easily skip the next subsection.

20. For simplicity, we present a theory in which the paradoxical sentences are exactly the sentences that are in the gap of a suitable minimal Kripkean fixed point (see Kripke, 1975). See Rossi $(2019,2021)$ for details on how to extend the theory to languages with richer vocabularies, in which further semantic notions, including revenge-breeding notions, can be formulated.

21. The extension of $Z$ is empty in the case of the least fixed point, and nonempty for a non-minimal fixed point.

22. In addressing the main objections faced by a bicontextualist treatment of agreement and disagreement, we also touch on more general issues regarding the theory of quantification adopted by bicontextualist semantics. For reasons of space, however, our discussion will be necessarily brief.

23. Here we are not yet concerned with the possibility that (6) may be paradoxical. We consider paradoxical sentences when discussing Nixon-Dean cases below.

24. We adopt in essence Glanzberg's reply to the objection from Nixon-Dean cases. The crucial difference between Glanzberg's theory and ours is that we limit the use of iterations of Kripke fixed-point models to paradoxical sentences.

25. The general objection is familiar and has been pressed by a number of authors, including e.g. Priest (2006, §§1.6-7) and Linnebo (2006, §4).

26. See e.g. Lewis (1991, pp. 61-68) and Williamson (2003, pp. 427-428). Relativists typically reject this objection, because it presupposes an absolutist interpretation of 'everything'. They insist that, once 'everything' is interpreted relativistically, the sentence is simply false, and hence inadequate to express their view.

\section{References}

Bacon, A. (2015). Can the classical logician avoid the revenge paradoxes? Philosophical Review, 124(3): 299-352.

Beall, J. (2009). Spandrels of Truth. Oxford University Press.

Feferman, S. (1991). Reflecting on incompleteness. Journal of Symbolic Logic, 56(1): 1-49.

Feferman, S. (2008). Axioms for determinateness and truth. Review of Symbolic Logic, 1(2): 204-217.

Field, H. (2008). Saving Truth from Paradox. Oxford University Press.

Friedman, H. and Sheard, M. (1987). An axiomatic approach to self-referential truth. Annals of Pure and Applied Logic, 33: 1-21. 
Gauker, C. (2006). Against stepping back: A critique of contextualist approaches to the semantic paradoxes. Journal of Philosophical Logic, 35 (4): 393-422.

Glanzberg, M. (2001). The liar in context. Philosophical Studies, 103(3): 217-251.

Glanzberg, M. (2004a). A contextual-hierarchical approach to truth and the liar paradox. Journal of Philosophical Logic, 33: 27-88.

Glanzberg, M. (2004b). Truth, reflection, and hierarchies. Synthese, 142(3): 289-315.

Glanzberg, M. (2015). Complexity and Hierarchy in Truth Predicates. In Achourioti, T., Galinon, H., Martinez Fernández, J., and Fujimoto, K., editors, Unifying the Philosophy of Truth, volume 36 of Logic, Epistemology, and the Unity of Science. Springer.

Halbach, V. (1999). Disquotationalism and infinite conjunctions. Mind, 108 (429): 1-22.

Halbach, V. (2011). Axiomatic Theories of Truth. Cambridge University Press. Horsten, L. (2012). The Tarskian Turn. Deflationism and Axiomatic Truth. MIT Press.

Kaplan, D. (1989). Demonstratives. In Almog, J., Perry, J., and Wettstein, H., editors, Themes from Kaplan. Oxford University Press.

Kaplan, D. and Montague, R. (1960). A paradox regained. Notre Dame Journal of Formal Logic, 1: 79-90.

Kripke, S. (1975). Outline of a theory of truth. Journal of Philosophy, 72: 690-716.

Lewis, D. (1991). Parts of Classes. Basil Blackwell.

Linnebo, O. (2006). Sets, properties, and unrestricted quantification. In Rayo, A. and Uzquiano, G., editors, Absolute Generality, pages 149-178. Oxford University Press.

McGee, V. (1985). A counterexample to modus ponens. The Journal of Philosophy, 82: 462-471.

Murzi, J. and Rossi, L. (2018). Reflection principles and the Liar in context. Philosophers' Imprint, 18(15): 1-18.

Murzi, J. and Rossi, L. (2020). Generalised revenge. Australasian Journal of Philosophy, 98(1): 153-177.

Myhill, J. (1960). Some remarks on the notion of proof. Journal of Philosophy, 57(14): 461-471.

Parsons, C. (1974). The Liar Paradox. Journal of Philosophical Logic, 3(4): 381-412.

Picollo, L. and Schindler, T. (2018). Disquotation and infinite conjunctions. Erkenntnis, 83(5): 899-928.

Picollo, L. and Schindler, T. (2019). Deflationism and the function of truth. Philosophical Perspectives, 32: 326-351.

Priest, G. (2006). In Contradiction. Oxford University Press, Expanded edition (first published 1987 Kluwer-Dordrecht).

Rayo, A. and Uzquiano, G. (1999). Toward a theory of second-order consequence. Notre Dame Journal of Formal Logic, 40(3): 315-325.

Rayo, A. and Williamson, T. (2003). A completeness theorem for unrestricted first-order languages. In Beall, J., editor, Liars and Heaps, pages 331-356. Oxford University Press. 


\section{Julien Murzi and Lorenzo Rossi}

Rossi, L. (2019). A unified theory of truth and paradox. The Review of Symbolic Logic, 12(2): 209-254.

Rossi, L. (2021). Bicontextualism. Unpublished manuscript.

Williamson, T. (2003). Everything. Philosophical Perspectives, 17(1): 415465 . 